\title{
Enhanced Recovery: Joining the dots
}

\section{Fawcett WJ, Mythen MG, Scott MJP}

Eight years ago, we wrote an editorial for this journal on Enhanced Recovery (ER) [1]. At that time, the concept of ER was over 15 years old (and had been practised under various names such as fast track surgery or accelerated recovery) and the uptake of it was sporadic. Yet even at that stage, it was recognised from established ER units that there were very large potential gains to be had for patients undergoing major elective surgery. Many of the benefits arose from the evidenced based, multimodal, multidisciplinary management of patients undergoing major surgery thereby minimizing the pathophysiological upset and the associated organ dysfunction. Historically, the easy-to-measure end point, length of hospital stay (LOS), was used to compare and judge the success of ER programmes. Whilst seemingly a useful, easy, binary measurement, it does have some merit, not least as remaining in a hospital environment is not risk-free, and associated with fasting, sleep disturbance, immobilisation and medication errors [2]. However, LOS is a very blinkered concept for assessing the efficacy of patient centred care. There are other described potential consequences for ERAS and the reduced stress response, including reduced complications and readmissions, improved cancer survival, cost, as well improving patient satisfaction and hastening return to preoperative function [3]. Finally, with over 10 million operations performed in the NHS annually and over 300 million worldwide (with both rising annually) [4] harnessing these potential gains from patients on ERAS pathways are enormous.

Since 2012, a wealth of data has been collected, and many of these proposed benefits have been realised. With good compliance to ERAS programmes, not only does LOS fall but readmissions are usually reduced or are unchanged (but importantly not increased which is a valid concern when attempting to reducing LOS)[5], although when readmissions do occur they are associated with, for example, ASA 3 or more and surgical duration in excess of 300 minutes in colorectal surgery[6]

The importance of reducing complications for surgical patients is well recognised as key area within healthcare systems on both short and long term outcomes [7]. It has been estimated that all cause postoperative deaths are the third biggest cause of mortality in the US 
following heart disease and cancer [8] The impact of non-fatal complications not only reduces patient satisfaction but may impact patient permanently in terms of disability free survival, functional recovery and health-related quality of life, with an enormous associated socioeconomic impact. ERAS pathways have consistently been shown to reduce both surgical and in particular medical complications, with ERAS pathway adherence an important factor in minimizing complications [3]. Very recently a large systematic review and meta-analysis of randomized controlled trials to examine perioperative prevention of postoperative pulmonary complications found that the most benefit was conferred by patients enrolled in ERAS pathways [9]. ERAS also confers procedure specific benefits (ie different benefits to different specialties) for example in joint replacement surgery, it is possible to demonstrate a reduction in postoperative delirium and cognitive dysfunction without the need for venous thromboembolism prophylaxis [10].

Early data supported the concept that ERAS patients may have improved disease-free survival for colorectal cancer patients [11], supported more recent data from the same group adding to the evidence: with good adherence to the ERAS pathways (>70\%), the risk of 5year cancer-specific death was lowered by $42 \%$ [12]. The factors here are multifactorial and may include reduction of perioperative stress, preoperative nutrition, prehabilitation, anaesthetic technique, reduction in complications, with earlier commencement of other therapies, such as chemotherapy.

ERAS has shown to be cost effective, in spite of seeming initially high (for example, reorganizing healthcare delivery, equipment and training minimal access surgery) with data supporting a sustained, but varied reduction in costs (up to $\$ 7,000 /$ patient reduction in direct cost) [13], with a return on investment of $\$ 3.8$ (range $\$ 2.4-\$ 5.1$ ) for every $\$ 1$ invested in ERAS [14]. However, even if LOS is reduced, it must be borne in mind that there may be significant post discharge spending [15].

In addition, ERAS has spread to a many other areas form the original four (colorectal, gynecology, musculoskeletal and urology) to practically every surgical specialty, including cardiac, thoracic, neurological, vascular, paediatric, head and neck, bariatrics and obstetrics [16]. ERAS has achieved marked successes in elderly patients [2]. The principles too have been applied to emergency general surgery [17] and there has also been great success globally, not only for higher income countries but low and middle income countries too [16] 
In spite of its many successes, ERAS still has a number of issues that need addressing in the future.

\section{Which programme and pathway for my patient?}

The advent of perioperative medicine pioneered at this College embraces and complements many themes of ERAS. Perioperative medicine itself has driven improvements and led to other initiatives such has Getting it Right First Time (GIRFT), Perioperative Quality Improvement Programme and National Emergency Laparotomy Audit (NELA). There are broadly overlapping principles to ERAS including strict pathway adherence with no variation, high quality data collection, audit and analysis to drive improvement. For example, PQIP includes Enhanced Recovery within its “Top 5 National Improvement Priorities for 2019-20" [18] and as a key pathway and the concept of as well as Dreaming (DRinking, EAting and Mobilising) is very similar to ERAS principles [19]. However, ERAS differs from many perioperative programmes in the close involvement of many members of the perioperative team: primary care, surgeons, nurses, pharmacists, physiotherapists, dietitians etc, as well as anesthetists [3].

\section{How many elements on the pathway?}

For some, the numbers of elements required in ERAS pathways - often in excess of twenty are seen as daunting [16]. This has generated a few practical issues, concerning both attaining compliance and which elements are really necessary. Whilst it is recognized that compliance and outcome are closely related, analogous to a dose response curve [11], it also recognized that not all components carry the same weight in terms of patient benefit. It is therefore logical that focus should be directed to those elements that confer the most benefits. In addition, given that some elements are viewed generic standards of care (such as avoidance of hypothermia, fluid excess, multimodal analgesia etc), this then may direct effort to more important areas. This area has recently been the subject of various papers and editorials in which two themes emerge. Firstly, the concepts described by Kehlet over 25 years ago and reiterated in a recent editorial, that his view that a return to the five early principles approach is required [20]. This includes the preoperative patient information, thoracic epidural anesthesia in open (but not laparoscopic) colonic surgery, avoidance of both fluid overload and hypovolemia, avoidance of a nasogastric tube and early oral feeding with mobilization. 
In addition, there has recently been focus on the importance of postoperative elements which although often difficult to implement are strongly associated with the greatest impact on optimal recovery. These include early removal and/or avoidance of urinary catheters and assistance with patient ambulation, and early feeding. [21]

\section{Why is implementation suboptimal?}

Another issue to be addressed concerns the barriers to both implementation of, subsequent adherence to, proven, evidenced based pathways. This concept - referred to as the "knowingdoing gap' continues to be a major obstacle in delivering ERAS [22]. These include patients, healthcare professional and institutional barriers, with many reasons such as ERAS programmes not meeting patient expectations and perspectives, issues related to medical and nursing staff (resistance to change, staff turnover and workload, inadequate training and support) and institutional reasons such as poor leadership, inadequate funding and importantly the lack of good data collection which will in turn not allow reliable auditing and implementation of continuous feedback [23].

\section{What is recovery?}

A paradox encompasses the term "Enhanced Recovery" inasmuch that the definition of recovery following surgery is not universal and it is only recently that due focus has centred in this crucial area. Classically, recovery has been divided into three phases. [24]. Familiar to all anaesthetists is the restoration of biological and physiological parameters such as adequate ventilation, blood pressure, oxygen delivery (if measured), urine output (if measured and more rarely used as guide to early patient management ) and temperature in the post anaesthetic care unit. The next phase revolves around a symptom-based approach to recovery treating pain, gastrointestinal function and the ability to perform basic activities prior to leaving hospital. Finally, possibly most importantly and definitely the most neglected in the past for patients, involves the resumption of full functional activities and Quality of life. This area has been the subject of much interest as it can take well over 6 months for patients undergoing colorectal surgery to return to baseline physical capacity [25], and in the latest PQIP report only $60 \%$ of patients resuming usual activities in this time frame[18]. There are many described measures of this, including Patient Reported Outcomes [26], although latterly The Quality of Recovery (QoR) scores also described by Myles' group have been widely used their description of 'days at home up to 30 days after 
surgery'(DAH 30) as a patient-centred outcome measure and is a easy to measure and useful marker of postoperative complications [27].

\section{Data Collection}

One issue that is seen as fundamental is good quality data collection. In order to achieve this contemporaneous good quality data collection and analysis is required, which can then be benchmarked against other centres. Where data is collected such as Enhanced Recovery after Surgery Interactive Audit System or PQIP (vide supra), valuable data is produced to drive change in the future. $[18,28]$.

\section{Research}

The issue of conducting high quality research in perioperative medicine is frustrated by the variation in practice between centres [29] often losing any potential signal in improvement. with the emphasis is thus moving towards much more tightly controlled patient-specific and procedure-specific interventions [30].

\section{The challenging patient}

We must recognize that a number of patients will not fit the usual postoperative trajectory. Whilst much of ERAS focusses on procedure specific issues, there are nevertheless patient specific issues who will pose a clinical challenge, for example an exaggerated stress response (both neuroendocrine and inflammatory responses). This area has been recently reviewed in this journal [31]. In particular the neuroendocrine response is affected by the relative expression of glucocorticoid and mineralocorticoid receptors, determined genetically, can be further modified by illness, age and deconditioning. These changes are associated with a number of common postoperative conditions, including cognitive dysfunction, myocardial injury, acute kidney injury, immunosuppression and infection, and muscle wasting, all of which will halt the expected progress of an ERAS patient [31] Other patients include those who are receiving long term high dose opioid or who are pain catastrophisers will need analgesic their pathways planned and modified in advance [29] 
In our last editorial, there was focus on the trimodal approach: analgesia, goal-directed fluid therapy (GDFT), and 'all the others'. The advances is these intraoperative areas are modest: analgesia is delivered on a procedure-specific basis[32], moving to away from central neuraxial blockade (in spite of good early pain control) due to associated problems of hypotension, immobility and need for urinary catheters. Although spinal anaesthesia has been shown to deliver low LOS and modify the physiological response [33,34] many are now using more peripherally sited blocks, combined with regularly administered multimodal analgesia. Whilst fluid balance endpoints are agreed - avoiding too little or too much and that the patients at highest risk and least cardiac reserve benefit the most - the ways of delivering this are debated. Many patients arrive relatively euvolaemic for theatre due to carbohydrate loading, reduced fasting and reduced need for bowel preparation and thus the main aims are to replace ongoing requirements and losses. Whilst other intraoperative areas are topical such as total intravenous anaesthesia (TIVA), deep neuromuscular blockade (DNB) and opioid-free anaesthesia (OFA) have produced some encouraging results, the use of TIVA (to reduce long term cancer recurrence), DNB (to allow reduction in intraabdominal pressures during minimally invasive surgery) and OFA (to reduce postoperative nausea and vomiting) have yet to find a clear, evidence based established place. An area of practice that requires renewed attention is ensuring that neuromuscular blockade is adequately reversed at the end of surgery, as the incidence is of postoperative residual curarisation (PORC) and the associated sequalae is still high, although it is reduced by the use of quantitative neuromuscular monitoring [35]. The focus for perioperative physicians will direct attention to other areas such as patient blood management, prehabilitation, perioperative opioid management to early recognition and management of postoperative organ dysfunction and perioperative opioid stewardship to minimize the risks of opioid-related harm.

\section{COVID-19}

The current pandemic has decimated our ability to carry out major elective surgery. Moreover, with reduced theatre capacity and bed availability, together with inevitable financial constraints that will face global economies, the scenario is set for ERAS to flourish. Some aspects of the ERAS pathway may have to be delivered remotely (such as preoperative and postoperative consultations and advice), the emphasis must be on delivering high quality care (including minimal access surgery where appropriate), all conducted in a safe environment of patient testing, isolation and wearing the correct PPE. 


\section{Joining the dots}

So how do we join the dots for the future? If the benefits outlined here from ERAS were a single intervention, such as a drug or a procedure, ERAS would represent probably the biggest advance in medicine for years, and its implementation would mandated. Yet for a multistep pathway, there will always be the temptation to bypass many of the elements, hoping for the same benefits. The future of ERAS lies perhaps not so much in tweaks to existing clinical pathways, but in rather more bold concepts:

- Defining what endpoint(s) constitute recovery

- Instituting and maintaining ERAS programmes in different specialties and healthcare systems

- Producing the best quality research and other clinical evidence to direct future clinical care pathways

- Producing the best quality institutional data to allow benchmarking, the incidence of complications and to direct local changes in practice

- Engagement of healthcare providers in future planning

To date, many dots have already been joined to create Kehlet's goal of "pain and risk-free operation"[36], but it is not time yet to put away our pencils. 


\section{REFERENCES}

1. Fawcett WJ, Mythen MG, Scott MJP. Enhanced Recovery - more than just reducing length of stay? British Journal of Anaesthesia 2012;109:671-674

2. Rasmussen LS, Jørgensen CC, Kehlet H. Enhanced recovery programmes for the elderly EJA 2016 European Journal of Anaesthesiology 2016;33:391-392

3. Ljungqvist $\mathrm{O}$, Scott M, Fearon KC. Enhanced recovery after surgery: a review. JAMA Surgery 2017;152:292-8.

4. Abbott TE, Fowler AJ, Dobbs TD, Harrison EM, Gillies MA, Pearse RM. Frequency of surgical treatment and related hospital procedures in the UK: a national ecological study using hospital episode statistics. British Journal of Anaesthesia 2017;119:24957.

5. ERAS Compliance Group. The Impact of Enhanced Recovery Protocol Compliance on Elective Colorectal Cancer Resection: Results From an International Registry. Annals of Surgery 2015;261:1153

6. Bennedsen AL, Eriksen JR, Gögenur I. Prolonged hospital stay and readmission rate in an enhanced recovery after surgery cohort undergoing colorectal cancer surgery. Colorectal Disease 2018;20:1097-108.

7. Khuri SF, Henderson WG, DePalma RG, Mosca C, Healey NA, Kumbhani DJ. Determinants of long-term survival after major surgery and the adverse effect of postoperative complications. Ann Surg 2005;242:326-41

8. Bartels K, Karhausen J, Clambey ET, Grenz A, Eltzschig HK. Perioperative organ injury. Anesthesiology 2013;119:1474-89

9. Odor PM, Bampoe S, Gilhooly D, Creagh-Brown B, Moonesinghe SR. Perioperative interventions for prevention of postoperative pulmonary complications: systematic review and meta-analysis. $B M J$ 2020;368:m540

10. Wainwright TW, Kehlet H. Fast-track hip and knee arthroplasty-have we reached the goal?. Acta Orthopaedica 2019;90:3-5

11. Gustafsson UO, Hausel J, Thorell A, Ljungqvist O, Soop M, Nygren J. Adherence to the enhanced recovery after surgery protocol and outcomes after colorectal cancer surgery. Archives of Surgery 2011;146:571-7.

12. Gustafsson UO, Oppelstrup H, Thorell A, Nygren J, Ljungqvist O. Adherence to the ERAS protocol is associated with 5-year survival after colorectal cancer surgery: a retrospective cohort study. World Journal of Surgery 2016;40:1741-7. 
13. Thiele RH, Rea KM, Turrentine FE, Friel CM, Hassinger TE, Goudreau BJ, Umapathi BA, Kron IL, Sawyer RG, Hedrick TL, McMurry TL. Standardization of care: impact of an enhanced recovery protocol on length of stay, complications, and direct costs after colorectal surgery. Journal of the American College of Surgeons. 2015;220:430-43.

14. Thanh NX, Chuck AW, Wasylak T, Lawrence J, Faris P, Ljungqvist O, Nelson G, Gramlich LM. An economic evaluation of the Enhanced Recovery After Surgery (ERAS) multisite implementation program for colorectal surgery in Alberta. Canadian Journal of Surgery. 2016:415-21

15. Bozic KJ,Ward L, Vail TP, Maze M. Bundled payments in total joint arthroplasty: targeting opportunities for quality improvement and cost reduction. Clin Orthop Relat Res. 2014;472:188-193

16. https://erassociety.org/guidelines/list-of-guidelines/ (Accessed July 2020)

17. Jordan LC, Cook TM, Cook SC, Dalton SJ, Collins K, Scott J, Peden CJ. Sustaining better care for patients undergoing emergency laparotomy. Anaesthesia 2020 (in press)

18. https://pqip.org.uk/pages/ar2019 (Accessed July 2020)

19. Levy N, Mills P, Mythen M. Is the pursuit of DREAMing (drinking, eating and mobilising) the ultimate goal of anaesthesia?. Anaesthesia. 2016;71:1008-12.

20. Kehlet H. ERAS implementation-time to move forward. Annals of Surgery 2018;267:998-9.

21. Aarts MA, Rotstein OD, Pearsall EA, Victor JC, Okrainec A, McKenzie M, McCluskey SA, Conn LG, McLeod RS. Postoperative ERAS interventions have the greatest impact on optimal recovery: experience with implementation of ERAS across multiple hospitals. Annals of Surgery 2018;267:992-7.

22. Kehlet H. Fast-track colonic surgery and the'knowing-doing'gap. Nature reviews Gastroenterology \& Hepatology 2011;8:539-40

23. Tanious MK, Ljungqvist O, Urman RD. Enhanced recovery after surgery: history, evolution, guidelines, and future directions. International Anesthesiology Clinics 2017;55:1-11.

24. Bowyer AJ, Royse CF. Postoperative recovery and outcomes-what are we measuring and for whom?. Anaesthesia. 2016;71:72-7.

25. Miller TE, Mythen M. Successful recovery after major surgery: moving beyond length of stay. Perioper Med 3, 4 (2014). https://doi.org/10.1186/2047-0525-3-4 
26. Kingsley C, Patel S. Patient-reported outcome measures and patient-reported experience measures. BJA Education 2017;17:137-44

27. Myles PS. More than just morbidity and mortality - quality of recovery and longterm functional recovery after surgery. Anaesthesia 2020;75: e143-150. 4.

28. Currie A, Soop M, Demartines N, Fearon K, Kennedy R, Ljungqvist O. Enhanced Recovery after Surgery interactive audit system: 10 years' experience with an international web-based clinical and research perioperative care database. Clinics in colon and rectal surgery 2019;32:075-81

29. Kehlet H. Enhanced postoperative recovery: good from afar, but far from good? Anaesthesia. 2020;75:e54-61

30. Joshi GP, Alexander JC, Kehlet H. Large pragmatic randomised controlled trials in peri-operative decision making: are they really the gold standard?. Anaesthesia 2018;73:799-803

31. Manou-Stathopoulou V, Korbonits M, Ackland GL. Redefining the perioperative stress response: a narrative review. British Journal of Anaesthesia 2019;123:570-83.

32. https://esraeurope.org/prospect/ (Accessed July 2020)

33. Levy BF, Scott MJ, Fawcett WJ, Rockall TA. 23-hour stay laparoscopic colectomy. Diseases of the Colon and Rectum 2009;52:1239-43

34. Day AR, Smith RVP, Scott MJP, Fawcett WJ, Rockall TA. Randomized clinical trial of spinal versus intravenous morphine on postoperative neuroendocrine responses. British Journal of Surgery 2015;102:1473-1479

35. Carvalho H, Verdonck M, Cools W, Geerts L, Forget P, Poelaert J. Forty years of neuromuscular monitoring and postoperative residual curarisation: a meta-analysis and evaluation of confidence in network meta-analysis. British Journal of Anaesthesia. 2020 (in press)

36. Kehlet H. Multimodal approach to control postoperative pathophysiology and rehabilitation. British Journal of Anaesthesia 1997;78:606-17 
